\title{
ON A MEAN VALUE INEQUALITY
}

\author{
BY ALBERTO TORCHINSKY ${ }^{1}$
}

Communicated by Alexandra Ionescu Tulcea, May 23, 1975

In this note we discuss a mean value inequality satisfied by functions $u(x, t)$ defined in the half space $R_{+}^{n+1}$ which are solutions of a partial differential equation of semielliptic type. We then apply this result to the study of spaces of nonisotropic Riesz potentials and to the determination of the classes which arise as traces of the functions $u(x, t)$. The justification for considering these functions lies in the fact that they are a natural substitute for harmonic functions when Laplace's equation is not satisfied and they are related to the study of singular integrals with mixed homogeneity. It is a pleasure to acknowledge the conversations we had with Dr. A. P. Calderón concerning these topics.

The mean value inequality. We let $\left\{A_{t}\right\}_{t>0}, A_{t s}=A_{t} A_{s}$ be a continuous group of affine transformations of $R^{n}$ leaving the origin fixed and denote its infinitesimal generator by $P$ so that $t(d / d t) A_{t}=P A_{t}$. We further assume that $(P x, x) \geqslant(x, x)$ for $x \in R^{n}$ and associate to each group $A_{t}$ a translation invariant distance function $\rho(x)$ defined to be the unique value of $t$ such that $\left|A_{t}^{-1} x\right|=1$, $\rho(0)=0$. To the transpose $A_{t}^{*}$ of $A_{t}$ we associate $\rho^{*}(x)$ in a similar fashion. As is well known $\operatorname{det} A_{t}=\operatorname{det} A_{t}^{*}=t^{\gamma}, \gamma=\operatorname{trace} P(\operatorname{see}[5, \S 1.1])$. For $\alpha=$ $\left(\alpha_{1}, \ldots, \alpha_{k}\right), 1 \leqslant \alpha_{i} \leqslant n$, and $x^{1}, \ldots, x^{k}$ in $R^{n}$ we let $\zeta=x^{1} \otimes \cdots \otimes x^{k}$ to be the element with components $\zeta_{\alpha}=\Pi_{i=1}^{k} x_{\alpha_{i}}^{i}$. For $n \times n$ matrices $A_{1}, \ldots, A_{k}$, we put $\left(A_{1} \otimes \cdots \otimes A_{k}\right)\left(x^{1} \otimes \cdots \otimes x^{k}\right)=A_{1} x^{1} \otimes \cdots \otimes A_{k} x^{k}$ and abbreviate this by $\otimes^{k} A x$ when $A_{i}=A, x^{i}=x$ for $1 \leqslant i \leqslant k$.

$\partial=\left(\partial / \partial x_{1}, \ldots, \partial / \partial x_{n}\right), \partial / \partial t$ and $\bigotimes^{k} A \partial$ acting on functions $u(x, t)$ have the obvious meaning. We set $p_{k}(t, \partial)=\bigotimes^{k} L A_{t}^{*} \partial$, where $L^{2}=\left(P+P^{*}\right) / 4 \pi$. Given a function $\psi(x)$ we define the dilations $\psi_{t}(x)=t^{-\gamma} \psi\left(A_{t}^{-1} x\right)$. A special role is played by $\varphi_{t}(x)$ with $\varphi(x)=e^{-\pi|x|^{2}}$. This particular function $\varphi_{t}(x)$ satisfies a differential equation, as is readily seen by taking Fourier transforms, namely $A \varphi_{t}(x)=0$ where

$$
\mathrm{A}=\frac{\partial}{\partial t}-\frac{1}{2 \pi t}\left(P^{*} A_{t}^{*} \partial, A_{t}^{*} \partial\right)=\frac{\partial}{\partial t}-\frac{1}{t}\left(L A_{t}^{*} \partial, L A_{t}^{*} \partial\right) .
$$

We also have $A u(x, t)=0$, whenever $u(x, t)=f_{*} \varphi_{t}(x), f \in S^{\prime}\left(R^{n}\right)$.

We now state the mean value inequality and give some applications in the following sections.

MEAN VAlUe INEQUALITY. Let $A u(x, t)=0$ and $0 \leqslant r \leqslant k$, then

AMS (MOS) subject classifications (1970). Primary 35 B45, 46E99; Secondary 35 K05.

${ }^{1}$ Research partly supported by NSF Grant GP 28251. 


$$
\left|p_{k}(t, \partial) u(x, t)\right|^{q} \leqslant c t^{-\gamma} \int_{t / 2}^{t} \int_{\rho(x-y) \leqslant t}\left|p_{r}(s, \partial) u(y, s)\right|^{q} d y \frac{d s}{s}
$$

for $1 \leqslant q<\infty$.

Nonisotropic Riesz potentials. (See [1], [3], [7], [12], [18] and [20].) For a positive real number $\alpha$ we define the Riesz potential $I_{\alpha}$ of order $\alpha$ of $f$ by means of

$$
\left(I_{\alpha} f\right)^{\wedge}(x)=\rho^{*}(x)^{-\alpha} \hat{f}(x), \quad 0<\alpha<\gamma,
$$

and for $1<p<\infty$, the classes $L_{\alpha}^{p}\left(R^{n}\right)=\left\{f \in L^{p}\left(R^{n}\right): f=I_{\alpha} \eta, \eta \in L^{p}\left(R^{n}\right)\right\}$ and we set $\|f\|_{p, \alpha}=\|f\|_{p}+\|\eta\|_{p}$.

We now consider the following variants of the Littlewood-Paley function to express the norm in $L_{\alpha}^{p}$ by an equivalent quantity (see [4], [10], [14], [16], [17]). Let

$$
G_{q}(k, \alpha, \lambda, x)=\left[\int_{0}^{\infty} \int_{R^{n}} \frac{\left|p_{k}(s, \partial) u(y, s)\right|^{q}}{(1+\rho(x-y) / s)^{\gamma \lambda}} s^{-\alpha q-\gamma} d y \frac{d s}{s}\right]^{1 / q}
$$

for $k \geqslant 1,0<\alpha<k$ and $\lambda>1$.

THEOREM. Let $u(x, t)=f_{*} \varphi_{t}(x)$; then $f$ is in $L_{\alpha}^{p}\left(R^{n}\right)$ if and only if $f$ is in $L^{p}\left(R^{n}\right)$ and $G_{2}(k, \alpha, \lambda, x)$ is in $L^{p}\left(R^{n}\right)$, provided $\lambda>2 / p$, and $\|f\|_{p, \alpha} \approx\|f\|_{p}+$ $\left\|G_{2}(k, \alpha, \lambda)\right\|_{p}$. Moreover, if $q \geqslant 2$ and $\lambda>q / p$, then $\left\|G_{q}(k, \alpha, \lambda)\right\|_{p} \leqslant c\|f\|_{p, \alpha}$, and if $\lambda=q / p$ and $p<2$ we have the weak-type inequality

$$
\left|\left\{x \in R^{n}: G_{q}(k, \alpha, q / p, x)>\mu\right\}\right| \leqslant c\|f\|_{p, \alpha}^{p} / \mu^{p} .
$$

That such weak-type inequalities follow from results in $[5, \S 3.3]$ was indicated to us by N. Aguilera.

Closely related to these questions are the functions $D_{q}^{\alpha}$ and $D_{p, q}^{\alpha}$ (see [2], [14], [15], [20]) defined as follows:

$$
\begin{aligned}
D_{q}^{\alpha}(x) & =\left[\int \frac{|f(x-y)-f(x)|^{q}}{\rho(y)^{\gamma+\alpha q}} d y\right]^{1 / q}, \\
D_{p, q}^{\alpha}(x) & =\left[\int_{0}^{\infty} \frac{1}{t^{\alpha q}}\left\{\int_{\rho(y) \leqslant 1}\left|f\left(x+A_{t} y\right)-f(x)\right|^{p} d y\right\}^{q / p} \frac{d t}{t}\right]^{1 / q}
\end{aligned}
$$

where $0<\alpha<1$ and $1 \leqslant p, q<\infty$.

Indeed we have the following result.

Theorem. Let $u(x, t)=f_{*} \varphi_{t}(x)$; then for $p>2 \gamma /(\gamma+2 \alpha)$,

$$
\|f\|_{p, \alpha} \approx\|f\|_{p}+\left\|D_{2}^{\alpha}\right\|_{p} \quad \text { and } \quad\left|\left\{x \in R^{n}: D_{2}^{\alpha}(x)>\mu\right\}\right| \leqslant c\|f\|_{p, \alpha}^{p} / \mu^{p}
$$

for $p=2 \gamma /(\gamma+2 \alpha)$.

Also if $1 \leqslant r \leqslant q<\infty, q \geqslant 2$ and $p>r \gamma /(\gamma+\alpha r)$, then 
$\left\|D_{r q}^{\alpha}\right\|_{p} \leqslant c\|f\|_{p, \alpha}, \quad$ and $\quad\left|\left\{x \in R^{n}: D_{r q}^{\alpha}(x)>\mu\right\}\right| \leqslant c\|f\|_{p, \alpha}^{p} / \mu^{p}$,

for $1<p=r \gamma /(\gamma+\alpha r)<2$.

Traces of the spaces $H^{\alpha, p}$. These results were obtained jointly with A. Ortiz and extend the interesting results of [6]. Let $0 \leqslant \alpha<1,1 \leqslant p<\infty$. We say that $u(x, t) \in H^{\alpha, p}$ if $A u(x, t)=0$ and

$$
|u|_{p, \alpha}=\sup _{x, t}\left[\frac{1}{t^{\gamma+\alpha p}} \int_{\rho(x-y) \leqslant t}\left[\int_{0}^{t}\left|p_{1}(s, \partial) u(y, s)\right|^{2} \frac{d s}{s}\right]^{p / 2} d y\right]^{1 / p}<\infty .
$$

Then the following holds. and

THEOREM. $u(x, t) \in H^{\alpha, p}$ if and only if $u(x, t)=f_{*} \varphi_{t}(x)$, where $f \in L_{\mathrm{loc}}^{P}\left(R^{n}\right)$

$$
\sup _{x, t}\left[\frac{1}{t^{\gamma+\alpha p}} \int_{\rho(x-y) \leqslant t}\left|f(y)-a v_{x, t} f\right|^{p} d y\right]^{1 / p}<\infty
$$

where

$$
a v_{x, t} f=\frac{1}{|\{z: \rho(z) \leqslant t\}|} \int_{\rho(x-y) \leqslant t} f(y) d y
$$

Therefore the spaces of functions $f(x)$ which arise as traces of functions $u(x, t)$ in $H^{\alpha, p}$ are global Lipschitz classes for $0<\alpha<1$ and BMO for $\alpha=0$.

\section{BIBLIOGRAPHY}

1. N. Aronszajn, F. Mulla and P. Szeptycki, On spaces of potentials connected with $L^{p}$ classes, Ann. Inst. Fourier (Grenoble) 13 (1963), 211-306. MR 31 \#5076.

2. R. J. Bagby, A difference quotient norm for spaces of quasi-homogeneous Bessel potentials, Studia Math. 40 (1971), 41-48. MR 47 \#802.

3. A.-P. Calderón, Lebesgue spaces of differentiable functions and distributions, Proc. Sympos. Pure Math., vol. 4, Amer. Math. Soc., Providence, R. I., 1961, pp. 33-49. MR 26 \#603.

4. - Algebras of singular integral operators, Proc. Sympos. Pure Math., vol. 10, Amer. Math. Soc., Providence, R. I., 1967, pp. 18-55.

5. A.-P. Calderón and A. Torchinsky, Parabolic maximal functions associated with a distribution. I, Advances in Math. 16 (1975), 1-64.

6. E. B. Fabes, R. L. Johnson and U. Neri, Spaces of harmonic functions representable by Poisson integrals of functions in BMO and $£_{p, \lambda}$, Univ. of Maryland Technical Report TR 74-44.

7. E. B. Fabes and N. M. Rivière, Symbolic calculus of kernels with mixed homogeneity, Proc. Sympos. Pure Math., vol. 10, Amer. Math. Soc., Providence, R. I., 1967, pp. 106-127. MR 38 \#5067. 137-193.

8. C. Fefferman and E. M. Stein, $H^{p}$ spaces of several variables, Acta Math. 129 (1972),

9. J. R. Hattemer, Boundary behavior of temperatures. I, Studia Math. 25 (1964/65), 111-155. MR 31 \#6064.

10. I. I. Hirschman, Jr., Fractional integration, Amer. J. Math. 75 (1953), 531-546. MR 15, 119.

11. W. R. Madych, On Littlewood-Paley functions, Studia Math. 50 (1974), 43-63.

12. P. I. Lizorkin, Nonisotropic Bessel potentials, Imbedding theorems for Sobolev space $L_{p}^{\left(r_{1}, \ldots, r_{n}\right)}$ with fractional derivatives, Dokl. Akad. Nauk SSSR $170(1966), 508-511=$ Soviet Math. Dokl. 7 (1966), 1222-1226. MR 34 \#3300.

13. J. Petree, On the theory of $£_{p, \lambda}$ spaces, J. Functional Analysis 4 (1969), $71-87$.

14. J. C. Polking, A Leibniz formula for some differentiation operators of fractional order, Indiana Univ. Math. J. 21 (1971/72), 1019-1029. MR 47 \#7414. 
15. C. Sadosky and M. Cotlar, On quasi-homogeneous Bessel potential operators, Proc. Sympos. Pure Math., vol. 10, Amer. Math. Soc., Providence, R. I., 1967, pp. 275-287.

16. C. Segovia and R. L. Wheeden, On certain fractional area integrals, J. Math. Mech. 19 (1969/70), 247-262. MR 39 \#7472.

17. C. Segovia and R. L. Wheeden, On the function $g_{\lambda}^{*}$ and the heat equation, Studia Math. 37 (1970), 57-93. MR 44 \#3124.

18. E. M. Stein, The characterization of functions arising as potentials, Bull. Amer. Math. Soc. 67 (1961), pp. 102-104. MR 23 \#A1051.

19. - Singular integrals and differentiability properties of functions, Princeton Math. Series, no. 30, Princeton Univ. Press, Princeton, N. J., 1970. MR 44 \#7280.

20. R. S. Strichartz, Multipliers on fractional Sobolev spaces, J. Math Mech. 16 (1967), 1031-1060. MR $35 \# 5927$.

21. R. L. Wheeden, $A$ note on a generalized hypersingular integral, Studia Math. 44 (1972), 17-26. MR 47 \#26.

22. A. Zygmund, Trigonometric series. Vols. I, II, 2nd ed., Cambridge Univ. Press, London and New York, 1968. MR 38 \#4882.

DEPARTMENT OF MATHEMATICS, CORNELL UNIVERSITY, ITHACA, NEW YORK $1 \mathbf{4}^{\prime} 850$

Current address: Swain Hall East, Indiana University, Bloomington, Indiana 47401 